\title{
Real-time Trust-Building Schemes for Mitigating Malicious Behaviors in Connected and Automated Vehicles
}

\author{
Dajiang Suo $^{1}$ and Sanjay E. Sarma ${ }^{1}$
}

\begin{abstract}
Vehicle-to-everything (V2X) communication has raised security and safety concerns. A malicious attacker can not only have a compromised vehicle broadcast spoofed positioning information to nearby vehicles, but also send fake emergency reports to traffic management systems. Previous works on mitigating such malicious behaviors focus on either developing cryptography mechanisms for identity authentication or conducting plausibility checking by individual vehicle to evaluate the trust of messages sent by others. This paper proposes two types of architectures (centralized and distributed) for mitigating malicious behaviors based on the collaboration between trust authorities and vehicles. They differ from previous approaches in that the trust authority and vehicle nodes collaborate but take different responsibilities in plausibility checking and maintaining trust in order to build trust on each vehicle in real-time, rather than only relying on the judgment from each individual vehicle. Also, the trust value maintained by the trust authority in our approach is for revoking certificates of vehicle nodes based on pre-defined patterns of trust changes. The pros and cons of each architecture are evaluated through an experimental simulation. In addition, the proposed architectures for trust-building can be extended in future works to encourage benign behaviors in the long run.
\end{abstract}

\section{INTRODUCTION}

Technologies that support V2X communication can bring benefits for vehicle operation and traffic management. However, this technological advancement also raises security and safety concerns over connected and automated vehicles (CAVs). Although cryptography schemes based on public key infrastructure have been developed for authenticating vehicle identities and ensuring the trustworthiness of CAVs, an inside attacker can still use compromised vehicles to initiate cyber-attacks. Specifically, an adversary can broadcast spoofed vehicle positions or speed through vehicle-to-vehicle (V2V) communications that jeopardize the safety of nearby vehicles. The adversary can also have compromised vehicles claim fake emergency events such as incidents or traffic congestion through vehicle-to-infrastructure (V2I) to make traffic management systems denial of service, as shown in Fig. 1 (bottom right). For other approaches built on plausibility checking on data, while providing more information for driving decisions such as braking or acceleration, they do not support safety-critical decisions from the traffic management perspective such as when to revoke certificates assigned to a given vehicle. Moreover, these approaches may not bring enough motivations for rationale attackers to behave benignly in the long run.

\footnotetext{
${ }^{1}$ Authors are with the department of Mechanical Engineering, Massachusetts Institute of Technology, Cambridge, MA 02139, USA djsuo, sesarma@mit.edu
}

This paper proposes two types of architectures (centralized and distributed) for mitigating malicious behaviors based on the collaboration between trust authorities and vehicles. These two architectures are derived from principles governing trust establishment and propagation in social networks. Also, they differ from previous approaches in that the trust authority and vehicle nodes collaborate to share responsibilities in plausibility checking and maintaining trust, rather than only relying on the judgment from each individual vehicle. Specifically, the trust authority is responsible for building and maintaining trust on each vehicle in real-time by checking the plausibility of V2X messages itself or delegate the tasks to vehicle verifiers. Also, the trust value maintained by the trust authority in our approach is for revoking certificates of vehicle nodes based on pre-defined patterns of trust changes. The pros and cons of each architecture are evaluated through an experimental simulation in the aspects of response capability, over-trust prevention, memory consumption and computation overload, and privacy etc.

The remainder of this paper is organized as follows: In section II, we describe previous works including algorithms of plausibility checking and trust models that have been developed for social networks; In section III, we present a brief description of vehicular networks, attacker's model and problem formulation; We discuss detailed design rationales in social networks and the process of building trust in section IV; In section V, we evaluate the two proposed architecture by simulation and discuss the pros and cons for each one.

\section{RELATED WORK}

Previous works on plausibility checking have mainly focused on evaluating the trustworthiness of data received by a single vehicle [1], [10], [9], [13], [18]. Leinmuller et al. propose algorithms based on fusing V2V information from a single vehicles or multiple ones to detect implausible position data. Ruj et al. define nine algorithms to detect implausible events contained in V2V messages [13]. In addition, multi-sensor fusion has been proposed that use information from on-board sensors (e.g., camera, Lidar) to determine if received V2V messages are malicious [11], [2], [21], [18].

There are also collaborative approaches in which vehicles share detection results of malicious behaviors [5], [17], [15]. $\mathrm{Yu}$ et al. [22] and Xiao et al. [20] propose multi-lateration methods to determine if a given vehicle really exists in a position it claims to be. Multiple nearby vehicles share each others detected ranges between the target vehicle and each 
of them. In addition, Schmidt et al. [15] suggest a scheme to aggregate information from multile vehicle sources.

For models of building trust in social networks, Jsang et al. propose a subjective logic to represent reputations of social entities and the propogation of trust [6], [7]. Zacharia and Maes [23] propose a model to manage the trust of each entity in on-line marketplaces such as Amazon and ebay, etc. Sabater and Sierra take a step further to incorporate multifacet evaluation of trust in their reputation model for social agents [14].

\section{SySTEM MODELS AND ASSUMPTIONS}

\section{A. Vehicular networks}

We assume that public-key infrastructure [24] is adopted to support inter-vehicle communications, that is, a trust authority is responsible for issuing and revoking certificates for authenticating vehicle identities. Fig. 1 provides an example of four stages of the life cycle of a connected vehicle. It should be addressed though that this is a generic description that helps readers understand security vulnerabilities in connected vehicles and may not be exactly the same as real-world deployment. We make reasonable assumptions based on previous publications and on-going test activities regarding connected vehicles in the U.S [12].

- Vehicle registration. The on-board unit (OBU) that restore credentials for $\mathrm{V} 2 \mathrm{X}$ communications will be preloaded with security management certificates [24] as initial credentials. This can be accomplished by original equipment manufactures (OEMs) for future connected vehicles. Currently in the U.S., for the connected vehicle pilot projects conducted by the department of transportation (DOT) [12], OBUs made by automotive suppliers are pre-loaded with credentials and will be installed in vehicles after drivers finish registration with the local authority.

- Joining vehicle networks. When a vehicle wants to join vehicular networks, it will use the security management certificates to acquire certificates and configuration parameters such as public and private key pairs. Since privacy-preserving property is out of the scope of this paper, we assume that a vehicle can only require a new certificate when the current one is expired. Admittedly, while providing trust authority (TA) with the ability to track vehicle behaviors within networks and revoke certificates when necessary, this design suffers privacy risks where a compromised TA track vehicle identity or even trajectory.

- V2X communications. During vehicle operation, a connected vehicle will use the key-pairs and certificates for encryption, decryption, signing and verifying V2X messages.

- Reporting malicious behaviors and revoking certificates. Each connected vehicle in the network can report behaviors that are suspected to be malicious to a TA. The latter entity will make decisions on certificate revocation based on these reports from multiple vehicles. In trustbased schemes, reports may include the evaluation of how trustworthy the target vehicle is. The TA may revoke certificates assigned to the target vehicle if the trust value on the target one is below a certain level.

\section{B. Attacker's model}

This paper focuses on inside attackers, that is, adversaries hold valid key pairs and certificates for V2X communication. For this reason, details of cryptographic mechanisms are not included in the description of internal designs of verifiers. We assume that attackers will actively spoof or tamper fake V2X messages and have compromised vehicles broadcast them to nearby vehicles or infrastructures.

There are different types of messages that an adversary can spoof, including dynamics information of the compromised vehicle such as position, speed and acceleration, and emergency reports like incidents, real-time traffic or severe weather conditions, etc. We use spoofing vehicle positions as an example to illustrate how malicious vehicles behave and trust-based schemes respond to such attacks. Fig. 2 shows four ways that an adversary can spoof or temper vehicle positions, which is proposed by Heijden et al [18]. Specifically, an adversary can spoof and have the compromised vehicle broadcast stationary (Fig. 2a) or random positions (Fig. 2c), or add constant offset (Fig. 2b) or random offset (Fig. 2d) to actual positions of the compromised vehicle. We should address though engineers should not take for granted that these are the only four ways that an inside attacker can spoof V2X messages or even just the content of vehicle dynamics although we use these four types of malicious behaviors as inputs in our experimental simulations.

\section{Problem formulation}

Before presenting our problem formulation, it is necessary to mention the format of the V2X messages we refer to in this paper. There are industry standards regulating the message format and exchange between vehicles and external entities. For example, SAE J2735 [8] defines the content and format of Basic Safety Messages (BSMs) used for V2V or V2I communication. For analysis purposes in the context of malicious behaviors in this paper, we only include message contents that are related to plausibility checking and trustbuilding without detailing cryptographic parameters such as key pairs or certificates in public-key infrastructure (PKI). Specifically, we define a V2X message as a tuple $\left(t, s_{t}, e_{t}\right)$ where $t$ represent the time that the message is generated, $s_{t}=\left\{x_{t}, v_{t}, a_{t} \ldots\right\}$ represents the vehicle state at time $\mathrm{t}$ including position, speed, acceleration, etc., $e_{t}$ represents internal (e.g., emergency braking) or external events (e.g., road congestion) that can influence the state of the target vehicle. Therefore, a message broadcasted by vehicle $v_{i}$ at time $t$ can be represented as $m_{t}^{i}=\left(v_{i}, s_{t}\right)$.

We define a claimer to be a vehicle $v_{i}$ that sends V2X messages $m_{t}^{i}$ with location claims and a verifier to be the $T A$ or a vehicle node $v_{j}$ that provides its evaluation of how trustworthy $v_{i}$ is, based on all $\mathrm{V} 2 \mathrm{X}$ messages it sent until current time step $\left\{m_{t}^{i} \mid t=1, \ldots, n\right\}$. All verifiers share their trust evaluation on $v_{i}$ to the TA. Then, the problem becomes 


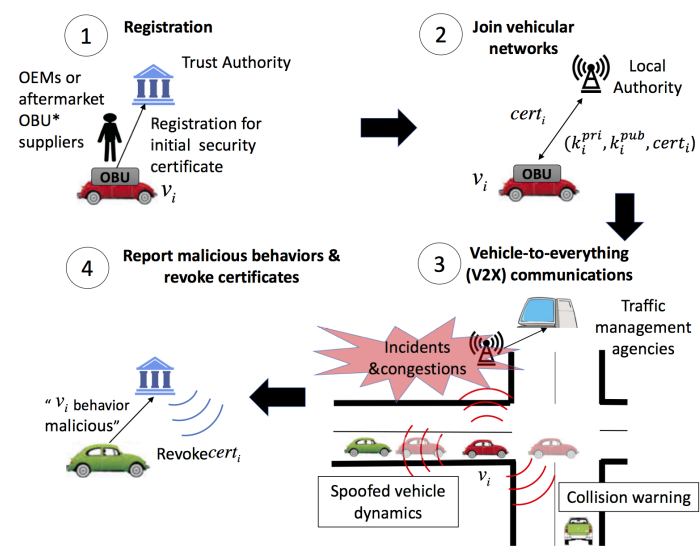

Fig. 1: A simplified life-cycle of connected and automated vehicles.

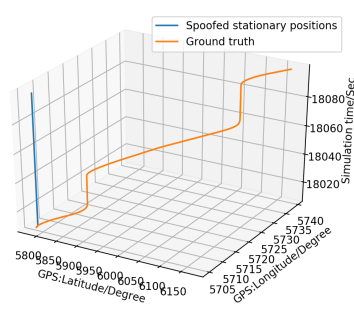

(a) Spoofing stationary positions

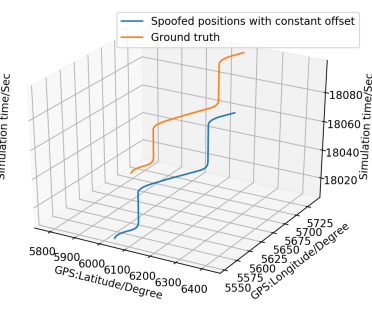

(b) Adding constant offset to actual positions

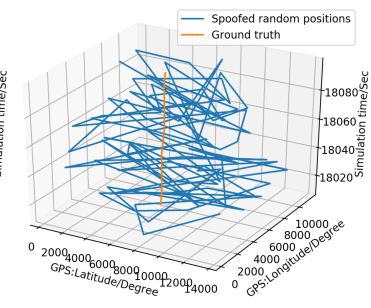

tions

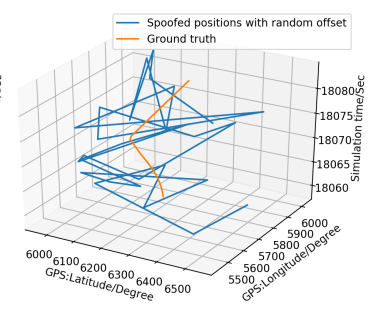

(d) Adding random offset to actual positions

Fig. 2: Time-space views of malicious behaviors: position spoofing [18]

how the TA can adjust its trust (evaluation) value on $v_{i}$ in real-time. As will be discussed in the next section, the TA becomes the only verifer by itself in centralized architecture while any vehicle can serve the role of verifers in distributed architecture.

\section{TRUST-BASED SCHEMES}

Many architectural decisions regarding trust-based schemes in vehicular networks are actually derived from principles governing social networks. Before presenting our system architectures for building trust on vehicles, we provide a brief overview of trust-building in social networks. We then describe the process and models for building trust on vehicles.

\section{A. An analogy between social and vehicular networks}

We start with three analogies between social networks and vehicular networks for the design rationales that we used in this paper, including paths of trust propagation, aggregation of opinions from different channels, and roles and reputations of intermediate nodes for indirect trust-building.

- Paths for trust propagation: In human society, a person can build trust on a given person through either direct interaction between them or based on indirectly recommendations from other persons, as shown in Fig. 3a [6]. For example, If a customer Alice chooses her host for lodging in on-line market places such as airbnb, she can build her trust on a given host Oscar either through her own experience of staying or get recommendations from Bob and Jack who have already stayed with Oscar before (Fig. 3a). Similarly, a TA can build trust on a given vehicle $v_{i}$ through direct communication or from indirect recommendations from vehicle verifiers (Fig. 3b).

- Aggregation of opinions from different aspects: as Sabater and Sierra suggest, reputation of a person or an entity can be evaluated from different social dimensions [14]. Take again the example of on-line market place of lodging. Alice may evaluate the reputation of the host Oscar from multiple angles including the rate, the cleanliness and the comfort of the room, whether the host is responsive and polite, etc. as shown in Fig. 3a. Similarly, in vehicular networks, a verifier can evaluate the trust of the vehicle $v_{i}$ by checking the plausibility of $v_{i}$ 's trajectory by using different rules [10], [15]. While one rule indicates malicious behaviors because it is implausible that any vehicle may always stay in the same place or move slowly even under smooth traffic flow, another rule may detect vehicles moving at a fast speed as malicious due to speed limits. In this regard, each module for plausibility checks contributes different weights to the evaluation of trust values on a given vehicle depending on the specific contexts.

- Roles and reputations of intermediate nodes: in the human society, people tend to trust a person who is recommended by someone who has good reputation. Similarly, a (vehicle) verifier who enjoys higher trust 


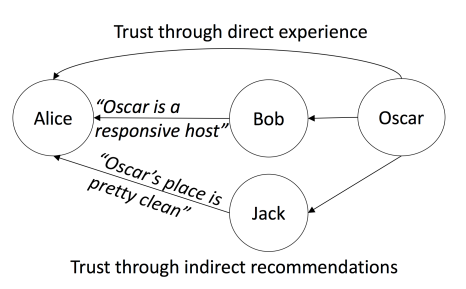

(a) Trust-building in on-line marketplaces

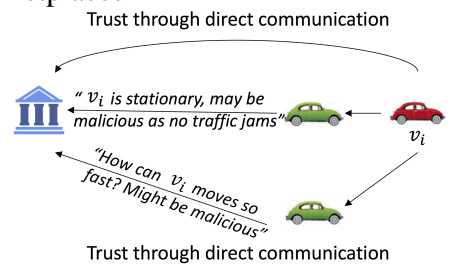

(b) Trust-building in vehicular networks

Fig. 3: An analogy of trust-building between social and vehicular networks

value (reputation) in the trust authority will have greater influence on the process of trust building. This aspect is reflected in Equation (1) proposed by Zacharia for calculating and updating trust values [23].

\section{B. Centralized vs. Distributed architectures}

We present two types of architectures for trust-building derived from principles in social networks. Design rationales for the centralized and distributed architectures are also discussed. In general, in the centralized architecture, the TA takes the role of verifier that directly checks V2V messages for building trust, as shown in Fig. 4a. On the other hand, the TA delegates the task of plausibility checking to vehicles in the distributed architecture, as shown in Fig. 4b. In the latter case, vehicles become actual verifiers who send their evaluation of trust on nearby vehicles to the TA.

In a centralized architecture, the TA monitors vehicle operation and interactions through V2X messages within a pre-determined region. V2X messages broadcasted by each vehicle is collected and then sent to the trust authority for verification (Fig. 4a upper-left). It should be addressed though that we ignore the infrastructure such as roadside units (RSUs) or digital sensors deployed on the road for collecting the V2X messages send by vehicles. In such design, the TA serves the role of authenticating vehicle identity and message integrity, checking the plausibility of the content of messages and calculating trust on the origin of a given message, as illustrated in Fig. 4a (bottom-left). The advantage of centralized architecture is that the TA has a broader view of each vehicle's behaviors in terms of time duration and distance of travels, which is useful in detecting malicious behaviors globally. However, this type of designs comes with the price of increased memory consumption of storing trajectory and computation overhead for conducting plausibility checks on each vehicle.
In a distributed architecture, the responsibility of verifying the plausibility of vehicle trajectory is delegated to vehicles. Fig. $4 \mathrm{~b}$ shows the detailed process of this. After evaluating the plausibility of claimed vehicle position or trajectory, a vehicle verifier will determine the trust value on the claimed vehicle and share the results to the trust authority. For this reason, the trust authority will only need to update its trust on the corresponding node. In addition to reducing memory consumption and computation overhead of the trust authority, another reason for adopting this design is to add more detection capabilities against local malicious behaviors such as spoofing a position that is out of the signal transmission range of vehicles [9]. However, the vehicle verifier may not be capable of detecting attackers in a global scale as the TA in the centralized architecture due to the ephemeral nature of vehicular networks. As Gerlach suggests, two vehicles that meet at current timestamp are not guaranteed to meet at the next [4]. In addition, distributed vehicles suffer the potential of over-trust and is more subjected to Sybil attacks, which will be discussed in the results of experimental simulations.

\section{Process of building trust}

In both centralized and distributed architecture, the internal data structure and algorithms that a verifier, either a TA or a vehicle, uses are similar. We first describe the process in details of how the TA in a centralized architecture builds trust on a given vehicle and then summarize the difference in internal designs between centralized and distributed architecture.

1) Initialization: The TA maintains two data structures in its memory: a trajectory table $\left\{\left(v_{i}, \operatorname{tr} j\left(v_{i}\right)\right): v_{i} \in V\right\}$ where $V$ is the set of registered vehicle ID and $\operatorname{trj}\left(v_{i}\right)$ represents the list of vehicle $v_{i}$ 's trajectory; a trust table $\left\{\left(v_{i}, \operatorname{trust}\left(v_{i}\right)\right): v_{i} \in V\right\}$ where $\operatorname{trust}\left(v_{i}\right)$ denotes the trust history of $v_{i}$ evaluated by TA. When a message $\left(v_{i}, t, s_{t}, e_{t}\right)$ is received from a new vehicle, the TA will establish corresponding entries $\operatorname{tr} j\left(v_{i}\right)$ and $\operatorname{trust}\left(v_{i}\right)$ for $v_{i}$ in the trajectory and trust tables respectively. $v_{i}$ will be also given an initial trust value $I$.

2) Plausibility checking: If the identity of the source node that sends a given V2X message $\left(v_{i}, t, s_{t}, e_{t}\right)$ can be authenticated, the verifier will extract the trajectory information of the source node $v_{i}$ from its trajectory table $\operatorname{tr} j\left(v_{i}\right)$ for plausibility checks. The plausibility checking module within a verifier can be regarded as a black box that takes as inputs the current message $\left(v_{i}, t, s_{t}, e_{t}\right)$ sent by $v_{i}$ 's and its trajectory $\left\{\left(n, x_{n}^{i}, y_{n}^{i}\right): n=1,2, \ldots t-1\right\}$ where $x_{n}^{i}$ and $y_{n}^{i}$ represent latitude and longitude coordinates of $v_{i}$ at $n^{\text {th }}$ time step, and outputs a value $T_{i, t}$ that represents the evaluation made by a verifier on the trust of $v_{i}$.

Designing plausibility checking for TA verifier in centralized architecture and a vehicle verifier in distributed architecture differs in three aspects: selecting detection algorithms, designing internal parameters for each algorithm, and deciding on the weight assigned to each algorithm (its contribution to the final evaluation score of trust on $v_{i}$ ). 


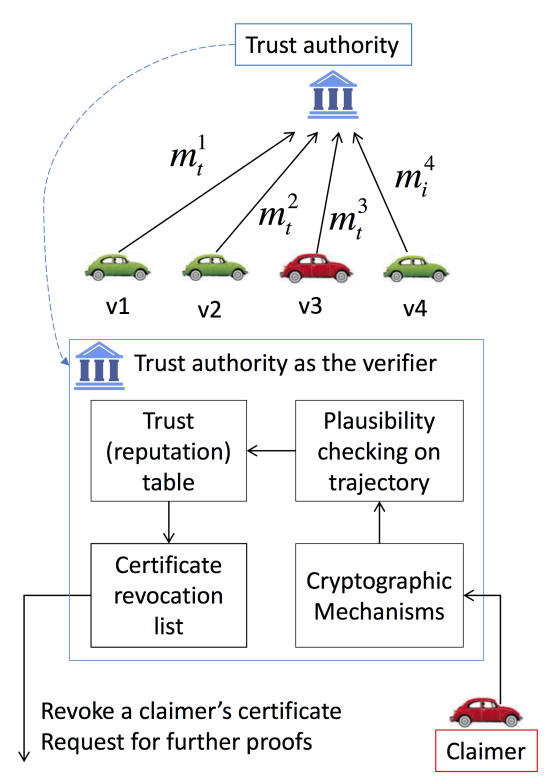

(a) The centralized architecture

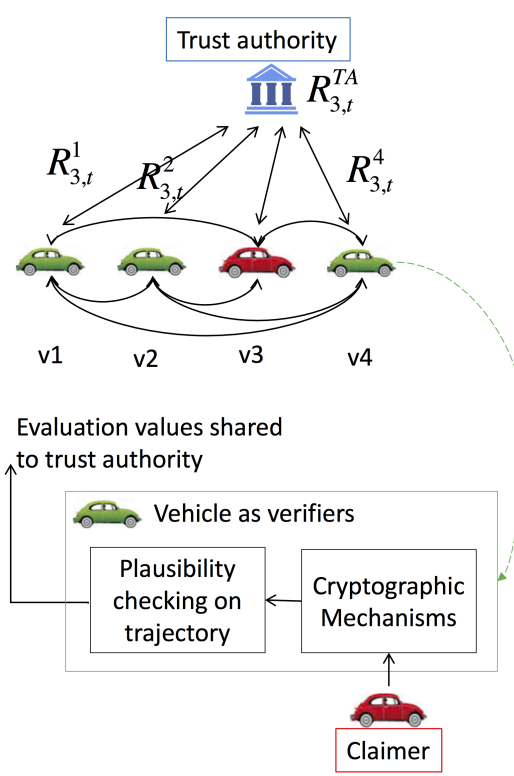

(b) The distributed architecture

Fig. 4: Two proposed architectures for trust-building

Algorithms used by the TA in centralized architecture can detect $v_{i}$ 's malicious behaviors in a global scale because the TA maintains and has real-time access to all observed positions along the trajectory of a given vehicle $v_{i}$. For example, the TA can use tracking algorithms such as Kalman filter [16] to track $v_{i}$ 's positions along the route of its travel and compare the predicted position with the position claimed by $v_{i}$ [1], [19]. Comparatively, a vehicle verifier in distributed architecture may not be capable of detecting malicious behaviors in a global scale. Due to the dynamic nature of vehicular networks, a vehicle verifer $v_{j}$ can only accumulate limited trajectory information of $v_{i}$ as "two vehicles that meet at current time stamp is not guaranteed to meet at the next." [4] However, $v_{j}$ may rely on the properties of the (radio) transmission signal such as radio propagation model to detect malicious behaviors in a local scale [9], [22]. For example, $v_{i}$ will be regarded as malicious if the position it claims is out of the transmission range (e.g., $<=300 \mathrm{~m}$ ) of signals received by $v_{j}$. Multiple vehicle verifier can even collaborate to check position claims based on multilateration principle [3] although this algorithm requires the sharing of observed positions of claimer vehicle $v_{i}$ among multiple verifiers.

Details of tuning internal variables for each algorithms are not the focus of this paper and can be found in [10], [9], [15], [19]. We also do not include a detailed discussion on how to determine weights for each algorithms although address that they can be changed dynamically depending on the specific context, as discussed in the analogy between social and vehicular networks.

3) Update trust: We adopt the model for updating trust value proposed by Zacharia [23] in his study of reputation systems for on-line community and make necessaries changes. Equation (1)-(3) [23] are adapted to the needs of trust-building in centralized architectures while Equation (4) [23] to the needs of distributed architecture.

In the centralized architecture, after the new trust value of vehicle $v_{i}$ is calculated based on results from plausibility checking, the final evaluation value $T_{i, t}^{T A}$ will be sent to the subcomponent for handling trust-building within TA, as shown in Fig. 4a (lower-left). It will then update its trust on $v_{i}$ by using (1). Equation (1) is adapted to the needs of trust-building in the centralized architecture. We keep the definition of parameters the same where $\theta$ represents the learning factor that decides how fast each claimer vehicle's trust in the TA changes after each iteration; $\Phi$ is called damping function which reflects the contribution to updated trust value on $v_{i}$ from its previous trust rating; D represents the maximum trust value that a vehicle can get and is different from Zacharia's original definition because TA will fully trust his own evaluation of $v_{i}$ 's trustworthyness; $E_{i, t-1}^{T A}$ is the normalized trust value of $v_{i}$ in previous time step which is defined in (3). Equation (2) defines the $\phi$ function where $\sigma$, the "forgetting factor" [23], decides the level of influence by previous trust value $R_{i, t-1}^{T A}$ on updated one $R_{i, t}^{T A}$.

$$
\begin{gathered}
R_{i, t}^{T A}=R_{i, t-1}^{T A}+\frac{1}{\theta} \Phi\left(R_{i, t-1}^{T A}\right) D\left(T_{i, t}^{T A}-E_{i, t-1}^{T A}\right) \\
\Phi\left(R_{i, t-1}^{T A}\right)=1-\frac{1}{1+\exp \left(\frac{-\left(R_{i, t-1}^{T A}-D\right)}{\sigma}\right)} \\
E_{i, t-1}^{T A}=R_{i, t-1}^{T A} / D
\end{gathered}
$$

In the distributed architecture, any vehicle verifier, say $v_{j}$, can send its evaluation of trust on claimer vehicle $v_{i}$ from $v_{j}$ 's internal plausibility checking, denoted as $T_{i, t}^{j}$, to TA. The TA will then update its trust on $v_{i}$ by using (4), as shown in Fig. 4b (lower-right). Note (4) differs from (1) 
TABLE I: Parameter choice in simulation

\begin{tabular}{|c|c|c|}
\hline \multicolumn{2}{|c|}{ Parameter name } & Values \\
\hline \multirow{3}{*}{$\begin{array}{c}\text { Parameters } \\
\text { for trust } \\
\text { calculation in (1)-(4) }\end{array}$} & $\theta$ & 20 \\
\cline { 2 - 3 } & $\sigma$ & 200 \\
\hline $\begin{array}{c}\text { Parameters } \\
\text { for algorithms } \\
\text { in plausibility } \\
\text { checking }\end{array}$ & $D$ & 3000 \\
\cline { 2 - 3 } & Min dist. moved & 5 \\
\cline { 2 - 3 } & Max speed difference & 25 \\
\cline { 2 - 3 } & Threshold for sudden appearance & 200 \\
\cline { 2 - 3 }
\end{tabular}

in that parameter $D$, the maximum range of trust value, is substituted with $R_{j, t-1}^{T A}$ that represents how much that TA trust verifier $v_{j}$ who shares its trust evaluation on $v_{i}$. This corresponds to the third principles of trust propagation in social networks that an agent's own reputation may influence how trustworthy that its recommendations are.

$$
R_{i, t}^{T A}=R_{i, t-1}^{T A}+\frac{1}{\theta} \Phi\left(R_{i, t-1}^{T A}\right) R_{j, t-1}^{T A}\left(T_{i, t}^{j}-E_{i, t-1}^{T A}\right)
$$

4) Revoke certificates: If vehicle $v_{i}$ is detected to be malicious, the TA can choose to revoke the certificate assigned to it. Then the question for designing a trust-building scheme becomes: given the trust history of $v_{i}$, when and how the TA should revoke certificates? We provide three strategies to illustrate the notion of trust-based certificate revocation although actual designs and implementations are contingent on engineers' choice.

- Assign a neutral trust value (e.g., $\mathrm{I}=\mathrm{D} / 2$ ) to $v_{i}$ and revoke its certificate when $v_{i}$ 's trust drops below a certain threshold

- Assign a low trust score (e.g., $\mathrm{I}=0$ or $\mathrm{D} / 10$ ) to $v_{i}$ and revoke $v_{i}$ 's certificate if its trust value start to decline at a certain point and the trend continues for a (predefined) period of time steps.

- In privacy-preserved scheme where every vehicle holds multiple short-lived certificates [24], mechanisms for checking the linkage between vehicles and its assigned certificates can be added to TA such that TA may revoke $v_{i}$ directly. Admittedly, this strategy can break the privacy

\section{EXPERIMENTAL EVALUATION}

\section{A. Simulation setup}

In this section, we evaluate two proposed architectures for trust-building on vehicle nodes by using simulation. We implement a discrete event simulator written in Python to emulate the process of $\mathrm{V} 2 \mathrm{~V}$ message exchange between different nodes. Specifically, for the trust-building module, we implement verifiers for TA in the centralized architecture and for vehicles in the distributed architectures based on the trust-building models discussed before. All parameters we used for calculating trust are summarized in Table I.

For the module of plausibility checking, we utilize four types of existing algorithms that are developed in [15], [10], [19], rather than developing our own as our focus is to compare centralized and distributed architectures to inform the design of trust-based schemes. There are two algorithms that are used by both TA and vehicle verifiers including: Minimum Distance Moved (MDM) [10] for checking whether a given vehicle has moved a threshold distance during a pre-defined interval and Simple Speed Check $(S S C)$ [19] which is a simplified Kalman Filter for checking the plausibility of vehicle movement based on estimation of maximum vehicle speed. Also, there are two algorithms we used are only for vehicle verifiers and they can only detect malicious behaviors locally: Acceptance Range Threshold (ART) [10] that can detect whether the claimed position of a given vehicle is out of the transmission range of signal (e.g., 300m) and Sudden Appearance Warning $(S A W)$ [10] whether a given vehicle appears suddenly in front of a vehicle verifier, indicating suspicious behaviors. If any of these algorithms within a given verifier has detected malicious behaviors, the corresponding verifier will output a low trust rating (0.1 in our implementation). Similarly, a verifer will output a high trust rating (0.9 in our implementation) if none of the algorithms above detect any malicious behaviors. It should be addressed that this is a simplified implementation of the plausibility checking module discussed in this paper as each module is supposed to be assigned to a weight that decides the contribution to final trust rating respectively, all parameters we selected for implementation is summarized in Table I.

In summary, there are three schemes that we evaluate in our python simulator and compare them based on four criteria. In particular, we take out of the two algorithms for detecting local malicious behaviors from the verifers as a benchmark for comparing centralized and distributed architecture.

- Scheme-1: Centralized architecture.

- Scheme-2: Distributed architecture with local detection algorithms.

- Scheme-3: Distributed architecture without local detection algorithms (i.e., $A R T$ and $S A W$ ).

The input dataset to our simulator is an open-source dataset-VeReMi-for evaluating algorithms for detecting malicious behaviors in vehicular networks [18]. VeReMi implements four types of malicious behaviors where attackers periodically or randomly sending malicious messages. This is illustrated with four examples shown in Fig. 2: broadcasting stationary positions (Fig. 2a), adding constant offset to actual positions of the claimer vehicle (Fig. 2b), broadcasting random positions in a given region (Fig. 2c), and adding random offset to actual position of the claimer vehicle (Fig. 2d). Note the attacker's behaviors appear to be less aggressive in the first two cases than in the last two cases in that the attacker changed claimed positions more abruptly and frequently in a short time duration. We choose data files corresponding to each of these malicious behaviors with two levels of traffic density (i.e., low with 35 vehicles and medium with 97 vehicles in total), which amounts to 8 rounds of simulations. The simulation time is approximately 25 seconds for low 
density and 100 seconds for medium density conditions.

\section{B. Results and discussions}

Both centralized and distributed architectures are tested against four types of malicious behaviors under low and medium density conditions, as shown in Fig. 5.

1) Capabilities of maintaining low trust for attacker nodes: The response capability we discuss here involves two aspects: first, how fast the TA can adjust its trust on a given vehicle when the vehicle starts to behave maliciously; second, whether the TA is able to keep the trust value of the malicious vehicles at a low level given different strategies an adversary take (e.g., sending spoof messages intermittently or gradually adding small perturbations to actual positions).

From the evaluation results, we can see that scheme-2 outperforms the first two schemes in almost all scenarios except under medium traffic density conditions (Fig. 5b and $5 \mathrm{~d}$ ), the trust value of attacker nodes in scheme- 2 are sometimes greater than in scheme-1. Not only does the TA can respond swiftly to malicious behaviors, but also maintain low trust scores for malicious vehicles. Attacker nodes fail to build up their reputations in the trust authority in Fig. 5a, 5c, $5 \mathrm{f}, 5 \mathrm{~g}$, and $5 \mathrm{~h}$. One reason may be that the vehicle verifiers in distributed architecture can detect malicious patterns in a local scale that are not visible to the trust authority in centralized architecture. One example is that local vehicle verifiers can decide if range between their position and the claimed position by the malicious node is greater than the transmission range threshold, as discussed before.

2) Vulnerability of distributed architecture in building trust: The poor performance of scheme-2 in Fig. $5 \mathrm{~b}$ and $5 \mathrm{~d}$ can be attributed to the ephemeral nature of vehicular networks. Indeed, it is not guaranteed that a vehicle (local) verifier in the distributed architecture can collect enough trajectory information of a given vehicle to determine its trustworthiness. Consider the scenarios that a malicious vehicle broadcasts (fake) stationary positions intermittently: it always broadcasts the same position for a couple of seconds (less than the detection threshold), wait for a while, and repeat again. If this happens, vehicle verifies in scheme-2 and 3 (distributed architecture) will not be able to respond to malicious behaviors. The same as malicious vehicles that add constant offset to their actual positions, as shown in Fig. $5 \mathrm{~b}$ and $5 \mathrm{~d}$. On the other hand, scheme- 1 performs better in attack scenarios with medium density traffic because the TA has a "global view" of vehicles' trajectory. Another possible explanation may be "over-trust" to which distributed architecture is vulnerable. Consider Fig. 5b and 5d again. The higher reputation of attacker nodes under scheme-2 (compared to scheme-1) may be because attackers first send regular messages to make (local) vehicle verifiers believe they are benign ones.

3) Memory, computation and communication overhead: Since the memory consumption and computation overhead incurred by trust calculation are negligible to storing and checking plausibility of vehicle trajectory, we only consider the latter one. We make three assumptions to facilitate the analysis: first, there are $n$ vehicles in the region monitored by the TA; second, each verifier, regardless of whether it is the TA or a vehicle, only keeps the most $m$ recent trajectory information for each vehicle it interacts with; third, the average running time of each algorithm for plausibility checking is $O(m)$. The third assumption is reasonable as the maximum time that a plausibility-checking algorithm takes to finish calculation corresponds to the length of the trajectory table. Furthermore, all algorithms for plausibility checking are running concurrently or the total number is much less than $m$, we can assume that the total running time of the plausibility checking module is also $O(m)$.

For the TA in centralized architecture, the memory consumption to store the trajectory table for all vehicles is $O(m n)$. The total running time of plausibility checking on trajectory of all vehicles is $O(m n)$ for fully sequential processing and $O(m)$ for fully parallel processing in the ideal case where the TA is fully aware of the expected number of vehicles in the networks. On the other hand, the TA in the distributed architecture only needs to store and update the trust value of each vehicles while the computation of plausibility checking are performed by vehicle verifiers.

For the communication overhead, scheme-1, 2 and 3 will have the same size of certificates, keys and signatures. However, scheme-1 suffers the overhead generated by transmitting vehicles' trajectory to the TA, while each vehicle only needs to provide its trust evaluations for neighbours in scheme- 2 and 3.

4) Privacy preserving property: Although privacy is not the focus of this paper, it is worth mentioning the potential conflict between security and privacy, which will be one of our future research directions. On one hand, a lot of design efforts in public key infrastructure is to add privacypreserving properties to certificates [24], including shared, short-lived and group signature, etc. On the other hand, that TA conducts plausibility checking and builds trust for each vehicle may break unlinkability of V2X messages. In other words, an adversary can link multiple messages with the origin vehicle such that he or she can track the vehicle. This can happen if a verifier, either a TA or a vehicle, is compromised. Obviously, the distributed architecture requires more protection from privacy breach because each vehicle verifier needs to link multiple messages with a given vehicle that sends these messages in order to evaluate its trust.

\section{CONCLUSIONS}

This paper presents a centralized and a distributed architecture for building trust on CAVs and evaluate their pros and cons through experimental simulation. The simulation result suggests that engineers should pay more attention to the lack of global views of vehicle behaviors by distributed architecture and its vulnerability in "over-trust". This issue can become more severe when multiple malicious node collude or create Sybil nodes. We will explore in future work how to build more robust mechanisms to defend against colluding or Sybil attacks. 


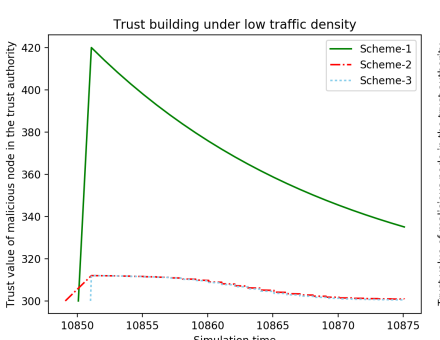

(a) Spoof stationary (b)
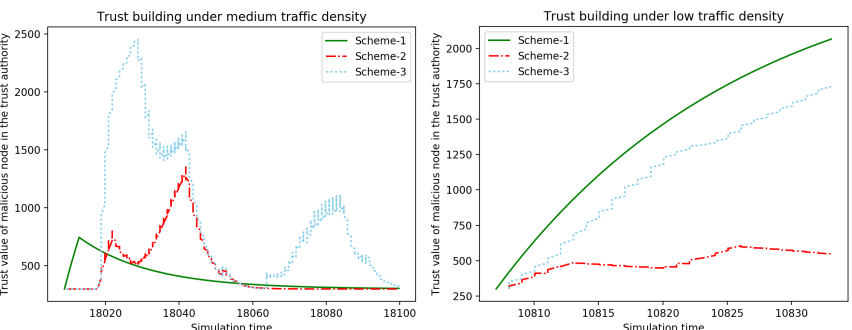

Spoof

(c) Add constant offset to actual positions:medium traffic density positions:low traffic density
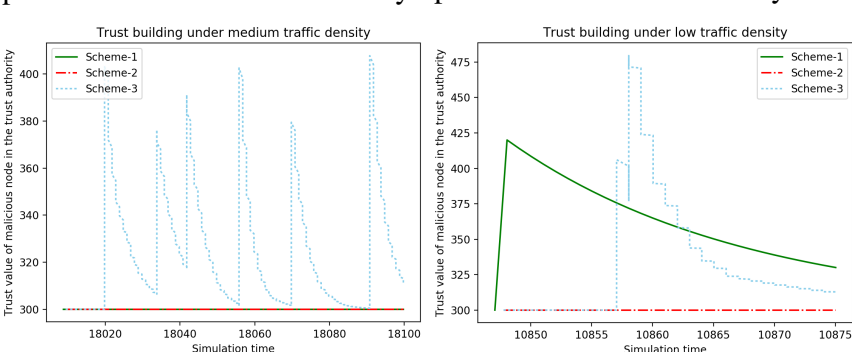

Spoof random (g) Add random offset to actua (f)

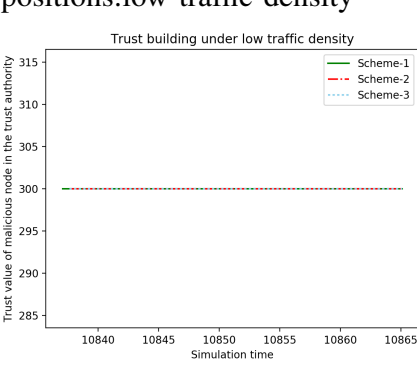

(e) Spoof random positions:low (f) traffic density

Fig. 5: Simulation results: real-time trust value of malicious vehicle in the trust authority

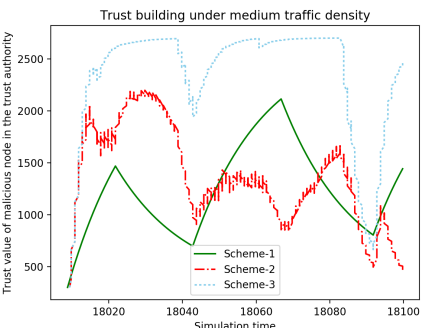

(d) Add constant offset to actual positions:medium traffic density

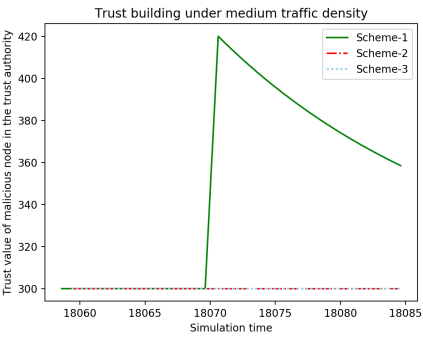

(h) Adding random offset to actual positions:medium traffic density

\section{REFERENCES}

[1] Rajesh P Barnwal and Soumya K Ghosh. Heartbeat message based misbehavior detection scheme for vehicular ad-hoc networks. In 2012 International Conference on Connected Vehicles and Expo (ICCVE), pages 29-34. IEEE, 2012.

[2] Norbert Bißmeyer, Sebastian Mauthofer, Kpatcha M Bayarou, and Frank Kargl. Assessment of node trustworthiness in vanets using data plausibility checks with particle filters. In 2012 IEEE Vehicular Networking Conference (VNC), pages 78-85. IEEE, 2012.

[3] Srdjan Capkun and Jean-Pierre Hubaux. Secure positioning of wireless devices with application to sensor networks. In IEEE infocom, number CONF, 2005.

[4] Matthias Gerlach. Trust for vehicular applications. In Eighth International Symposium on Autonomous Decentralized Systems (ISADS'07), pages 295-304. IEEE, 2007.

[5] Zhen Huang, Sushmita Ruj, Marcos A Cavenaghi, Milos Stojmenovic, and Amiya Nayak. A social network approach to trust management in vanets. Peer-to-Peer Networking and Applications, 7(3):229-242, 2014.

[6] Audun Jøsang, Ross Hayward, and Simon Pope. Trust network analysis with subjective logic. In Proceedings of the 29th Australasian Computer Science Conference-Volume 48, pages 85-94. Australian Computer Society, Inc., 2006.

[7] Audun Jøsang, Stephen Marsh, and Simon Pope. Exploring different types of trust propagation. In International Conference on Trust Management, pages 179-192. Springer, 2006.

[8] John B Kenney. Dedicated short-range communications (dsrc) standards in the united states. Proceedings of the IEEE, 99(7):1162-1182, 2011.

[9] Tim Leinmüller, Christian Maihöfer, Elmar Schoch, and Frank Kargl. Improved security in geographic ad hoc routing through autonomous position verification. In Proceedings of the 3rd international workshop on Vehicular ad hoc networks, pages 57-66. ACM, 2006.

[10] Tim Leinmuller, Elmar Schoch, and Frank Kargl. Position verification approaches for vehicular ad hoc networks. IEEE Wireless Communications, 13(5):16-21, 2006.

[11] Marcus Obst, Laurens Hobert, and Pierre Reisdorf. Multi-sensor data fusion for checking plausibility of $\mathrm{v} 2 \mathrm{v}$ communications by visionbased multiple-object tracking. In 2014 IEEE Vehicular Networking Conference (VNC), pages 143-150. IEEE, 2014.

[12] Department of Transportation. Connected Vehicle Pilot Deployment Program, 2019 (accessed April 15, 2019).
[13] Sushmita Ruj, Marcos A Cavenaghi, Zhen Huang, Amiya Nayak, and Ivan Stojmenovic. On data-centric misbehavior detection in vanets. In 2011 IEEE Vehicular Technology Conference (VTC Fall), pages 1-5. IEEE, 2011.

[14] Jordi Sabater and Carles Sierra. Regret: reputation in gregarious societies. In Agents, volume 1, pages 194-195, 2001.

[15] Robert K Schmidt, Tim Leinmüller, Elmar Schoch, Albert Held, and Günter Schäfer. Vehicle behavior analysis to enhance security in vanets. In Proceedings of the 4th IEEE Vehicle-to-Vehicle Communications Workshop (V2VCOM2008), 2008.

[16] Sebastian Thrun, Wolfram Burgard, and Dieter Fox. Probabilistic robotics. MIT press, 2005.

[17] Seyhan Ucar, Sinem Coleri Ergen, and Oznur Ozkasap. Data-driven abnormal behavior detection for autonomous platoon. In 2017 IEEE Vehicular Networking Conference (VNC), pages 69-72. IEEE, 2017.

[18] Rens W van der Heijden, Thomas Lukaseder, and Frank Kargl. Veremi: A dataset for comparable evaluation of misbehavior detection in vanets. In International Conference on Security and Privacy in Communication Systems, pages 318-337. Springer, 2018.

[19] Rens Wouter Van der Heijden. Misbehavior detection in cooperative intelligent transport systems. PhD thesis, Universität Ulm, 2018.

[20] Bin Xiao, Bo Yu, and Chuanshan Gao. Detection and localization of sybil nodes in vanets. In Proceedings of the 2006 workshop on Dependability issues in wireless ad hoc networks and sensor networks, pages 1-8. ACM, 2006

[21] Gongjun Yan, Stephan Olariu, and Michele C Weigle. Providing vanet security through active position detection. Computer communications, 31(12):2883-2897, 2008.

[22] Bo Yu, Cheng-Zhong Xu, and Bin Xiao. Detecting sybil attacks in vanets. Journal of Parallel and Distributed Computing, 73(6):746756, 2013.

[23] Giorgos Zacharia and Pattie Maes. Trust management through reputation mechanisms. Applied Artificial Intelligence, 14(9):881-907, 2000.

[24] Tao Zhang and Luca Delgrossi. Vehicle safety communications: protocols, security, and privacy, volume 103. John Wiley \& Sons, 2012 . 